\title{
Dispersion through an obstacle array: a wind tunnel investigation
}

\author{
V. Garbero ${ }^{1,2}$, P. Mejan ${ }^{1}$, R. Perkins ${ }^{1}$, P. Salizzoni ${ }^{1,2}$ \\ \& L. Soulhac ${ }^{1}$ \\ ${ }^{1}$ LMFA, Ecole Centrale de Lyon, France \\ ${ }^{2}$ DIASP, Politecnico of Turin, Italy
}

\begin{abstract}
In order to study flow and dispersion of a passive scalar through a group of obstacles, we performed wind tunnel experiments in a neutrally stratified boundary layer. The aim of the study was to evaluate the influence of obstacle geometry and of wind direction on the spreading of a passive scalar plume. An idealized urban neighborhood was simulated by means of an array of aligned blocks, whose height $\mathrm{H}$ was approximately $1 / 16$ of the simulated boundary layer depth; the width and the length of each block were $5 \mathrm{H}$. The pollutant source was placed at height $\mathrm{H} / 2$ within the array. Flow-field and mean concentration data were obtained for different wind directions, which varied from 0 to $45^{\circ}$, and for different spacing between the obstacles, which varied from $\mathrm{H}$ to $2 \mathrm{H}$; all measurements were performed within and above the obstacle array. Measurements of concentration have been examined in order to evaluate the mass exchange processes and their dependence to the wind orientation. Our goal was to verify the extent of the applicability of a simple analytical model in rather complex geometries.
\end{abstract}

Keywords: atmospheric dispersion, urban pollution, obstacle array, flow field.

\section{Introduction}

The study of plume dispersion in urban environment, because of its complexity, is not generally solvable by computational means and so it has been conducted by using physical models in wind tunnel or field experiments. Investigations are usually focused on dispersion through idealized arrays of simple patterns, in order to facilitate a general understanding of the overall plume behaviour and its dependence by the most relevant parameters (Britter and Hanna [1]). 
A key parameter in determining the plume dispersion behaviour is the plan area density of the buildings, $\lambda_{p}=W L /((S+W)(S+L))$, related to the spacing between the obstacles and their height (Macdonald et al. [2]); three different aerodynamic flow regimes occur in any arrays, depending on the building density: the isolated roughness flow $\left(\lambda_{p}<10 \%\right)$, the wake interference flow (10 $\left.\%<\lambda_{p}<17 \%\right)$ and the skimming flow $\left(\lambda_{p}>15-20 \%\right)$.

Davidson et al. [3] investigated the near-field plume behaviour emitted by point sources in relatively sparse array of cubes and verified an approximated Gaussian distribution for the concentration profiles. Macdonald et al. [4] examined the effect of obstacle width-to-height ratio on plume dispersion around urban buildings in a wind tunnel and at a field site; they found that the measured concentration profiles exhibited a Gaussian distribution profile. Differently, Theurer et al. [5] showed that, in more irregular arrays of buildings, Gaussian plume model may approximate concentration distribution only in far-field, i. e. for distance larger than a radius of homogenization, while in near-field dispersion depends strongly on the local arrangements of the obstacles and the assumption of a Gaussian plume gives erroneous results.

In our work we studied the problem of dispersion through a dense group of obstacles and looked at the effects of wind direction and obstacle layout pattern on the spreading of a passive scalar plume.

\section{Experimental setting}

The problem of plume dispersion in urban areas has been investigated by means of wind tunnel experiments, performed in the Wind Tunnel at the Laboratoire de Mecanique des Fluides et Acoustique in Lyon. The wind tunnel working section was $14 \mathrm{~m}$ long $\times 2.5 \mathrm{~m}$ high $\times 3.7 \mathrm{~m}$ wide and a neutrally stratified urban boundary layer of approximately $0.8 \mathrm{~m}$ was generated at scale $1: 400$. A $1.5 \mathrm{~km} \times 1.5 \mathrm{~km}$ urban neighborhood was simulated by an aligned configuration of building-like obstacles with dimensions $\mathrm{H}=50 \mathrm{~mm}$ and $\mathrm{W}=\mathrm{L}=250 \mathrm{~mm}$, laying out on a $3.7 \mathrm{~m} \times 3.7 \mathrm{~m}$ rotating plate of the working section. The ratio of the height of the obstacles to the depth of the boundary layer is $1 / 16$, ensuring a somewhat similar condition between the simulated and the real case. The reference free stream velocity $U_{\infty}$ at the boundary layer height was set at $5 \mathrm{~m} / \mathrm{s}$, yielding a characteristic Reynolds number of $1.5 \times 10^{4}$ that assured an asymptotic behavior of the turbulent flow, independent of the Reynolds number.

Mean wind speed and turbulence intensity were measured upwind the obstacle array, to verify they were consistent with typical urban flow profiles. Flow field measurements above the group of obstacles were performed with conventional hot-wire anemometry, a technique which did not allow to measure velocity and turbulence intensity within the obstacles; Laser Doppler Anemometry was adopted to replace it.

Concentration measurements were made by detecting a passive gas tracer (ethane) discharged from a punctual source, which released the gas with a negligible discharge momentum: a Flame Ionisation Detector was used to record vertical 

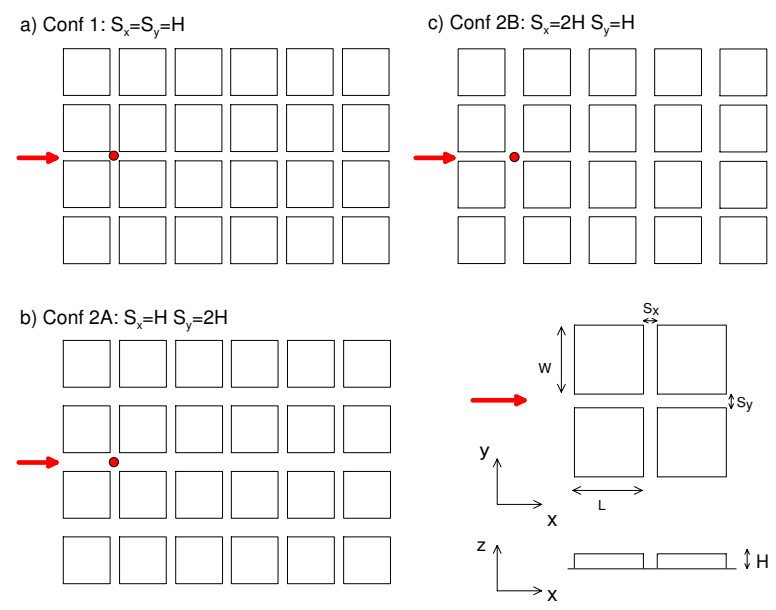

Figure 1: Geometry of the obstacle arrays studied, $\mathrm{W}=\mathrm{L}=5 \mathrm{H}$. (a) Conf 1: $S_{y}=S_{x}=\mathrm{H}$; (b) Conf 2A: $S_{x}=\mathrm{H} S_{x}=2 \mathrm{H}$; (c) Conf 2B: $S_{x}=2 \mathrm{H}$ $S_{x}=\mathrm{H}$.

and horizontal mean concentration profiles, within and above the obstacle array.

The measured concentrations were non-dimensionalised by introducing a new variable $\mathrm{K}$ :

$$
K=\frac{10^{-6} C U_{H} L H}{Q}=\frac{10^{-6} C U_{H} 5 H^{2}}{Q}
$$

where $\mathrm{C}$ is the measured mean concentration in ppm, $\mathrm{Q}$ is source strength in $\mathrm{m}^{3} \mathrm{~s}^{-1}, \mathrm{H}$ is the height of the obstacles, $\mathrm{L}$ is their length and $U_{H}$ is the upwind velocity at height $\mathrm{H}$.

The basic experimental configuration, shown in Fig. 1(a), consisted of the mentioned obstacles aligned parallel to the mean wind direction and equally spaced at a distance $\mathrm{S}=\mathrm{H}=50 \mathrm{~mm}$; the characteristic plan area density was $\lambda_{p}=69 \%$. Other two different configurations were studied: one, shown in Fig. 1(b), consisted of aligned obstacles spaced along the y-axis at a distance $S_{y}=2 \mathrm{H}$ and along the X-axis at a distance $S_{x}=\mathrm{H}$; the other, drawn in Fig. 1(c), was characterised by $S_{y}=\mathrm{H}$ and $S_{x}=2 \mathrm{H}$. The plan area density was the same for the two configurations, $\lambda_{p}=59 \%$, so that it was possible to extrapolate on one hand the influence of the package density and on the other hand the effect of the geometry on the plume spreading through and above the group of buildings.

In order to study how the wind direction affects the dispersion, the turning surface on which the group of buildings laid was rotated of various angles: the obstacle layout $S_{y}=S_{x}=\mathrm{H}$ was studied for wind incident angles respect to the $\mathrm{X}$-axis equal to $12.5,27.5$ and $47.5^{\circ}$.

The source was placed at the center of an intersection at height $\mathrm{H} / 2$ within the obstacle array. Concentration measurements were performed along the $y$-axis 
within the array, at height $\mathrm{H} / 2$, and above it, at height $2 \mathrm{H}$, for different rows downwind the source; our goal was to evaluate how the lateral spreading of the plume evolves with distance from the source and how it was influenced by the local arrangement of the array. Vertical concentration profiles were also measured, in order to estimate the vertical plume spreading development.

\section{Result}

\subsection{Profiles of mean concentration within the canopy: array configuration}

Macdonald et al. [4] and Davidson et al. [3] showed that the mean concentration field through an obstacle array can be modelled satisfactorily by suitably adapted Gaussian profiles; they suggested the measured concentration distributions may be fitted to a Gaussian profile laterally,

$$
K_{y}=K_{y o} \exp \left\{\frac{\left(y-y_{c}\right)^{2}}{2 \sigma_{y}^{2}}\right\}
$$

and to a reflected Gaussian profile vertically,

$$
K_{z}=K_{z o} \exp \left\{\frac{\left(z-z_{c}\right)^{2}}{2 \sigma_{z}^{2}}+\exp \frac{\left(z+z_{c}\right)^{2}}{2 \sigma_{z}^{2}}\right\}
$$

In the equation, the lateral and vertical plume standard deviations $\sigma_{y}$ and $\sigma_{z}$ represent the dispersion parameters, $y_{c}$ is the distance to the centreline of the plume and $z_{c}$ the effective vertical height. The values of these parameters were not estimated by fitting the theoretical profiles to the measured profiles, as the other authors did, but they were calculated as moments of the measured $K_{y}$ and $K_{z}$, by using the following relations:

$$
\begin{aligned}
& \bar{y}=y_{c}=\frac{\int y K(y) d y}{\int K(y) d y} \sigma_{y}^{2}=\frac{\int(y-\bar{y})^{2} K(y) d y}{\int K(y) d y} \\
& \bar{z}=z_{s}=\frac{\int z K(z) d z}{\int K(z) d z} \quad \sigma_{z}^{2}=\frac{\int(z-\bar{z})^{2} K(z) d z}{\int K(z) d z}
\end{aligned}
$$

Fig. 2 shows measured concentration profiles, in the variables obtained by the previous equations, together with the theoretical Gaussian profile; three different configurations of obstacle array, obtained by varying the spacing-to-height ratio, i.e. $S_{x}=S_{y}=\mathrm{H}\left(\right.$ Conf 1), $S_{x}=\mathrm{H} S_{y}=2 \mathrm{H}($ Conf $2 \mathrm{~A})$ and $S_{x}=2 \mathrm{H} S_{y}=\mathrm{H}$ (Conf 2B), were studied for one wind direction, that is parallel to x-axis. The horizontal profiles of mean concentration referred to $S_{x}=2 \mathrm{H} S_{y}=\mathrm{H}$ configuration, represented by diamond in Fig. 2, agree with the theoretical Gaussian profile, plotted in solid line; the horizontal profiles measured in the other two layouts show a Gaussian profile but they do not appear self-similar. Specially, they seem to be fitted by two different Gaussian distributions, plotted in dashed lines; the narrower one describes the concentration values within the channel shaped by two block 

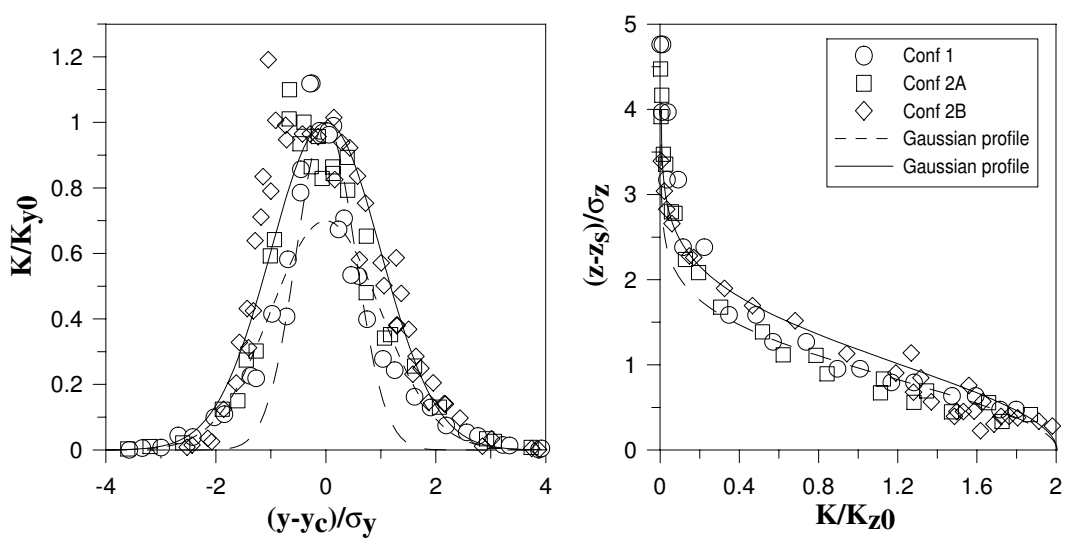

Figure 2: Horizontal and vertical profiles of mean concentration for three obstacle configuration, measured at several locations downstream of the source.

lines, the larger one fits the concentration values behind the obstacles. A similar behaviour can be explained by an important effect of channelling, that limits the dispersion mechanisms in the lateral direction, owning to the presence of recirculation regions in the lee of the obstacles: it indicates a typical skimming flow regime.
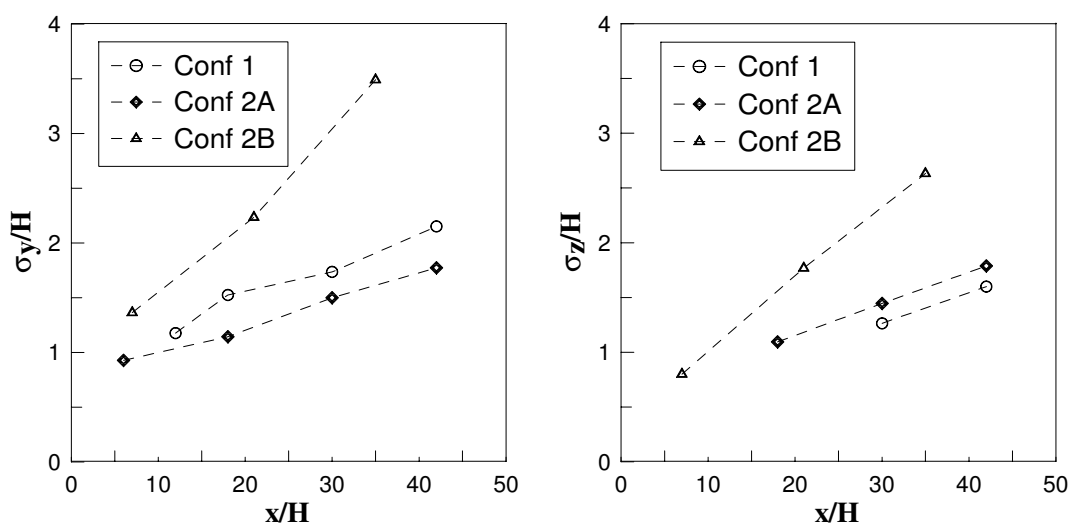

Figure 3: Lateral and vertical plume spread as a function of distance from the source in obstacle arrays with various spacing-to-height ratio.

In order to study the effect of the array geometry on the plume spreading, we plot the $\sigma_{y}$ and $\sigma_{z}$ values for the three different configurations. Fig. 3 shows that 

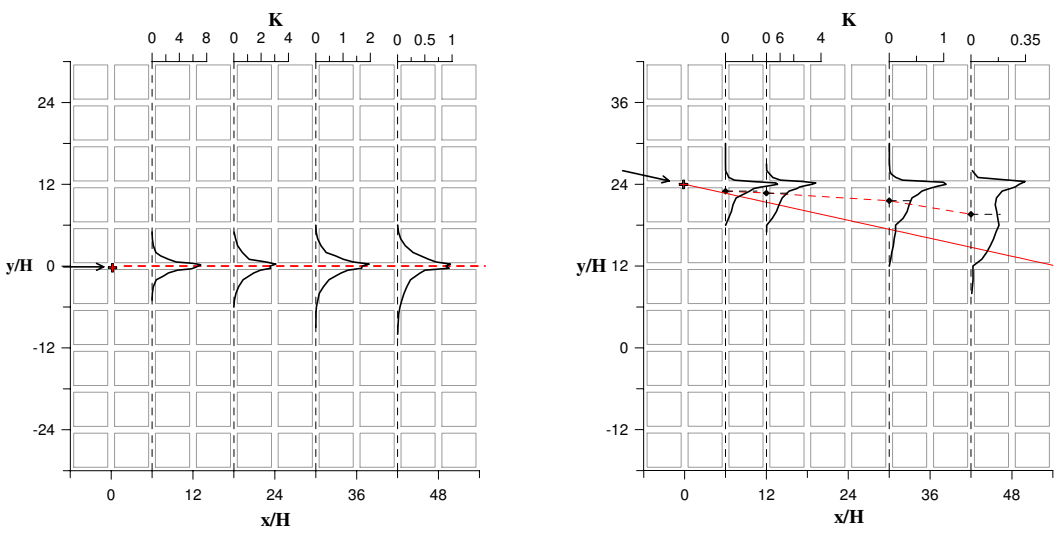

Figure 4: Plume behaviour for various wind direction: (a) parallel to $\mathrm{x}$-axis; (b) $12.5^{\circ}$ respect to $\mathrm{X}$-axis.

the plume behaviour is similar for Conf1 and Conf2A, while it seems quite different for Conf 2B. The augmented width of the longitudinal channels, i.e. $S_{y}=2 \mathrm{H}$, doesn't affect the general behaviour of the plume, whereas the augmented width of the lateral streets, i.e. $S_{x}=2 H$, produces larger values of lateral and vertical plume standard deviations, that indicates an enhanced dispersion. Presumably, a wake interference regime is established and the vortices induced by obstacles interact with the mean flow in the longitudinal channels, by enhancing the lateral mass transport, which in turn affects the plume structure and smooths the concentration profiles.

\subsection{Profiles of mean concentration within the canopy: wind direction}

While a Gaussian model may describe the plume dispersion through the canopy with a normal wind, it is no more able to represent the concentration distributions within the array with various incident wind angle. In order to better describe how the wind direction affects the plume behaviour through an array, we have shown in Fig. 4 and 5 the evolution of the horizontal concentration profiles with distance from the source for a wind parallel to the $x$-axis, inclined of $12.5^{\circ}, 27.5^{\circ}$ and $47.5^{\circ}$ respect to the $\mathrm{x}$-axis.

The channelling mechanism is evident in Fig. 4 (a): the plume appears to be confined into the channel within which it is released, inhibiting the spread of the plume into neighboring channels. Coherent turbulent structures arise into the channels and limit the mass exchange in the lateral direction, which mainly relied on the mechanism of intermittency.

In Fig. 4(b) and 5(a), the effect of the channelling on the plume behaviour is shown: the centreline is shifted with respect to the expected straight line, parallel to the wind direction and passing through the source. As noted by Theurer [5], 

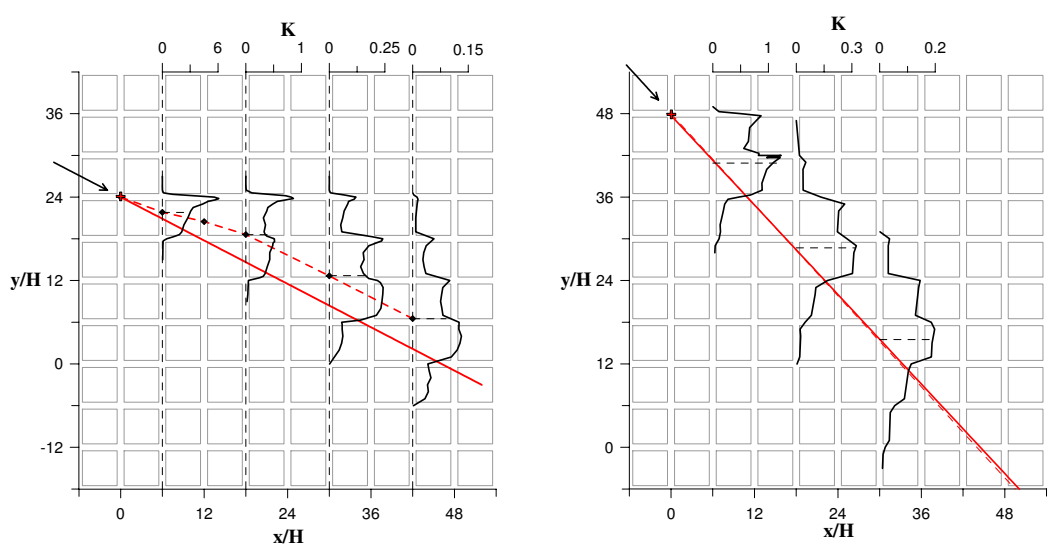

Figure 5: Plume behaviour for various wind direction: (a) $27.5^{\circ}$ respect to $\mathrm{x}$-axis; (b) $47.5^{\circ}$ respect to $\mathrm{X}$-axis.
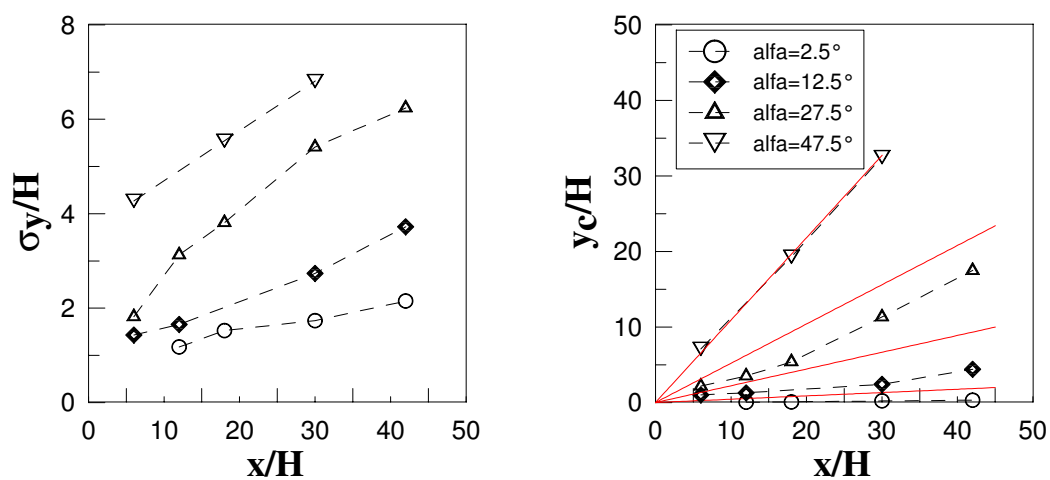

Figure 6: Lateral plume spread and lateral offset as a function of distance from the source in obstacle array by varying mean wind direction respect to the $\mathrm{x}$-axis.

wide obstacles deflect the plume for angles of the wind on the front row up to 30 degrees and the plume does not follow the direction of the overhead mean wind. Nevertheless, this effect vanishes for a wind incident angle respect to $\mathrm{x}$-axis of $50^{\circ}$ : as it is showed in Fig. 5(b), the lateral offset of the plume is in agreement with the value predicted by the mean wind orientation.

In Fig. 6 statistics from the measured profiles are plotted, in order to evaluate how the plume behaviour is affected by the wind orientation. The evolution of the plume centreline $y_{c}$ as function of distance from the source confirms what we explained before on the effect of channelling. In regard to the lateral standard deviation, as the incident angle of the flow increases respect to the $\mathrm{x}$-axis, a steeper 


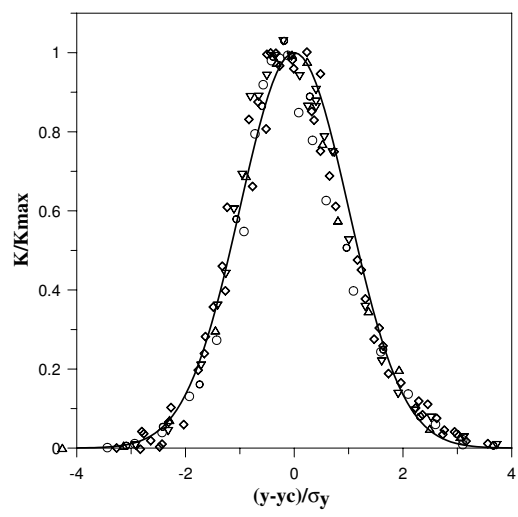

Figure 7: Horizontal profiles of mean concentration for all the obstacle configurations, measured at the $5^{\text {th }}$ row from the source.

slope characterizes the growth of $\sigma_{y} / H$ as function of $\mathrm{x} / \mathrm{H}$; it indicates a quicker and larger spreading.

\subsection{Profiles of mean concentration above the canopy}

In order to analyse how the disposition of the obstacles influences the plume development above the canopy, concentration measurements have been performed at height $2 \mathrm{H}$ from the terrain for all the configurations. In Fig. 7 horizontal profiles of mean concentration confirm that the mean concentration field can be modelled satisfactorily by adapted Gaussian profiles; it demonstrates that the inhomogeneity of the horizontal concentration profiles, due to the local arrangement of the obstacles, vanishes at height $2 \mathrm{H}$.

Nevertheless, the evolution of lateral standard deviation $\sigma_{y} / H$ and lateral offset $y_{c} / H$ as a function of the downwind distance from the source is similar to that within the canopy (Fig. 8): the dispersion rate strongly depends on the topography, the shape and the disposition of the obstacles, within and above the canopy.

\section{Conclusion}

The effects of obstacle geometry and wind direction on plume dispersion within and above an urban canopy have been examined in wind tunnel experiments.

Three different configurations with various spacing-to-height ratios have been studied for a wind direction parallel to the x-axis and different phenomena have been observed: (1) for $S_{x} \leq S_{y}$ values, an important effect of channelling was observed, which inhibited the plume lateral spread and limited the mass exchanges; (2) for $S_{x}=2 S_{y}$ values, the vortex structures induced by obstacles interacted with the mean flow in the channels, enhancing the lateral mass transfer and the mixing 

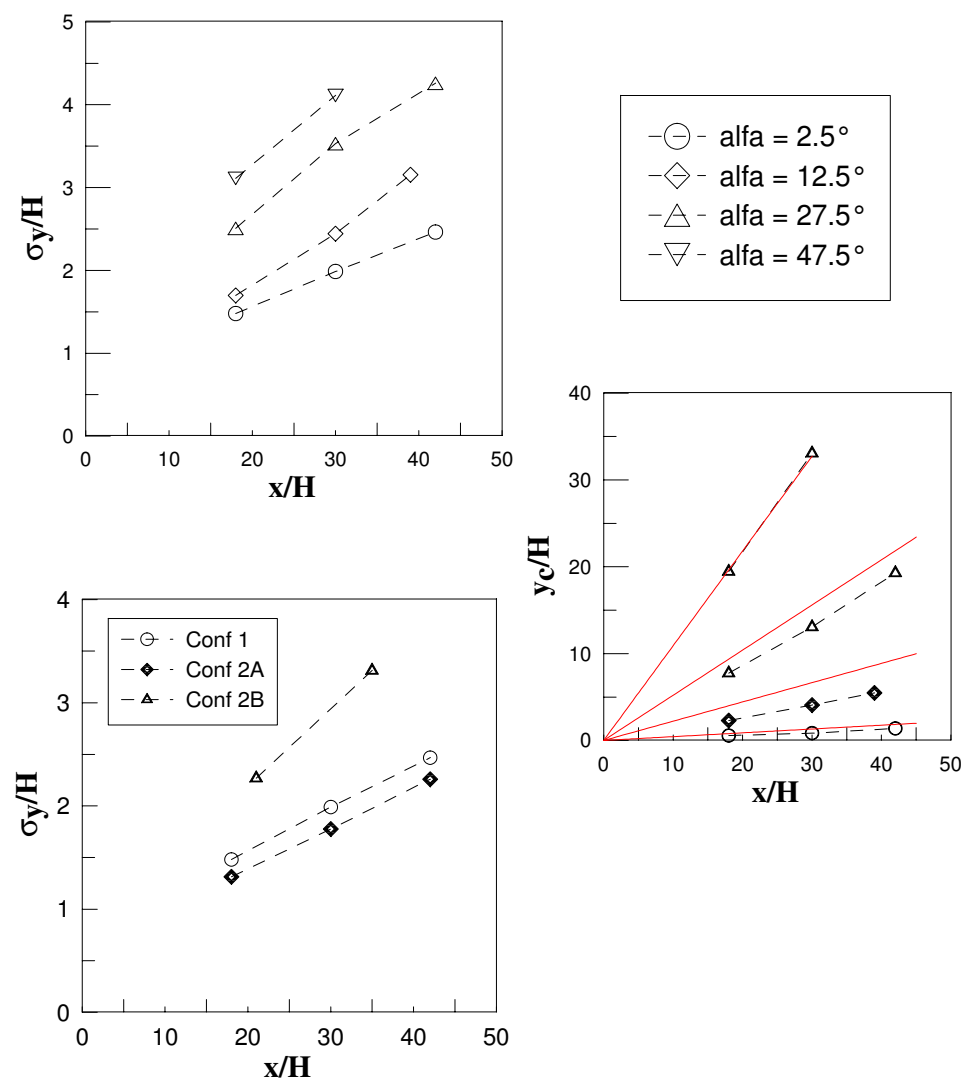

alfa $=2.5^{\circ}$

alfa $=12.5^{\circ}$

alfa $=27.5^{\circ}$

alfa $=47.5^{\circ}$

Figure 8: Lateral plume spread and lateral offset as a function of distance from the source in obstacle array with various mean wind and various spacing-toheight ratio.

within the array.

Mean concentration measurements have been conducted through the $S_{x}=S_{y}=$ $\mathrm{H}$ array for various wind incident angles and non-Gaussian distributions were observed; the directionality introduced by the presence of the streets don't agree with the isotropy of a Gaussian profile. Besides, the wind channelling is a relevant phenomenon for wind incident angle $<30^{\circ}$, since the plume centreline is not determined by the direction of the overhead mean wind, but it is affected by the obstacle layout.

Measurements of mean concentration have been performed above the canopy and may be fitted by adapted Gaussian distributions; the inhomogeneity of the horizontal distributions, due to the local obstacle arrangement, disappear at height $2 \mathrm{H}$ and the dispersion may be described by analytical models. 


\section{References}

[1] Britter, R.E. \& Hanna, S.R., Flow and dispersion in urban areas. Annual Review of Fluid Mechanics, 35, pp. 469-496, 2003.

[2] Macdonald, R.W., Griffiths, R.F. \& Cheah, S.C., Field experiments of dispersion through regular arrays of cubic structures. Atmospheric Environment, 31(6), pp. 783-795, 1997.

[3] Davidson, M.J., Snyder, W.H., Lawson, R.E. \& Hunt, J.C.R., Wind tunnel simulations of plume dispersion through groups of obstacles. Atmospheric Environment, 30(22), pp. 3715-3731, 1996.

[4] Macdonald, R.W., Griffiths, R.F. \& Hall, D.J., A comparison of results from scaled field and wind tunnel modelling of dispersion in arrays of obstacles. Atmospheric Environment, 32(22), pp. 3845-3862, 1998.

[5] Theurer, W., Plate, E.J. \& Hoeschele, K., Plume dispersion through large groups of obstacles - a field investigation. Atmospheric Environment, 30(21), pp. 3245-3256, 1996. 